These pages offer you the chance to open topics for debate, discuss the latest issues, or even offer advice to fellow readers. One letter in this issue calls for dentists and dental technicians to work closer together. Send us your views on this or anything to do with working in the dental profession. We look forward to hearing from you.

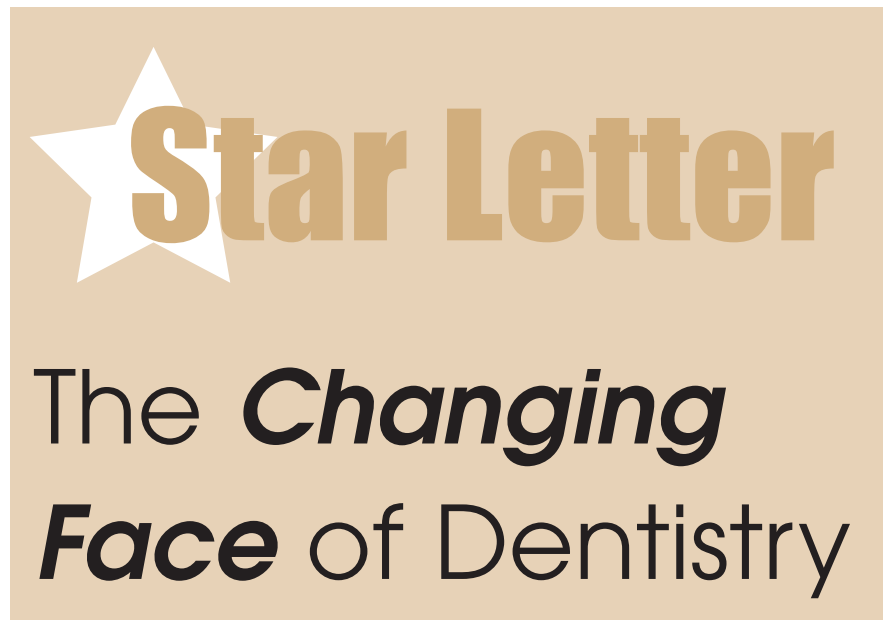

The role of Professionals Complementary to Dentistry (PCDs) has changed quite radically in recent times and further changes are likely in the foreseeable future. Both hygienists and therapists now have increased clinical responsibilities, but perhaps one of the most interesting developments of late, has been the emergence of jointly qualified dental hygienist/therapists, or oral health practitioners which may be an appropriate designation for those holding a dual qualification. These skilled individuals, who have expertise in periodontal, preventive and restorative care, are equipped to undertake much of the routine dentistry for both the child and adult population, thus allowing the dentist to focus on more specialised work. Given the apparent shortage of dentists throughout the UK, these professionals are in a position to make a significant contribution to the delivery of oral healthcare, and undertake procedures that historically, have been in the domain of the dentist.
In comparison with medicine, dentistry has lacked vision in terms of patient care. Medicine has developed to the extent of now having nurse practitioners, specialists and consultants and indeed, in more remote and rural areas of the country, much of routine medical care is undertaken by individuals who have received appropriate training. Perhaps the clinical role of PCDs should be extended further to improve the service to the patient. The need for hygienists and oral health practitioners to work from a prescription is a hindrance, as is the need for a dentist to be on the premises whilst certain procedures are being done. The benefits of being able to administer inferior dental block analgesia to a patient are obvious, but the need for a dentist to be on-site is less clear. The role of the dental nurse could also be expanded significantly to allow them, again following appropriate training, to undertake more clinically involved procedures in line with nurses in medicine. Examples of these could be impression taking, placement of temporary restorations, suture removal and even administration of local analgesia.

The modernisation of dentistry throughout the UK dictates that the existing workforce takes into account the substantial contribution which can be made by PCDs in addressing the unacceptable levels of disease that exist in many parts of the country. These are exciting times in the education and career development of PCDs, and we should embrace the change and associated challenge that lies ahead for all involved in patient care!

\section{Margaret Ross,}

President, British Dental Hygienists' Association

\title{
Let me climb the career ladder
}

A great piece by Arveen Bajaj (Dental nurse to dentist in one easy move? Feature, Spring 05) and another great issue. Keep it up.

I am acutely aware of how difficult it is to access an undergraduate course in dentistry having recently applied to three dental schools. I have qualified as a dental technician, dental nurse, dental hygienist and most recently as a therapist and I hoped my next step would be dental school.

After applying to the several schools I did the relevant PCD (Professionals Complementary to Dentistry) training but I did not even get past the UCAS application system. I have no A levels but hoped my training would get me in. Sadly none of my qualifications held any academic weight which is truly outrageous. This seems nonsensical because when I applied to do a masters degree I was accepted.
A modular training programme seems like a great opportunity for PCDs to progress academically and more importantly clinically. I firmly

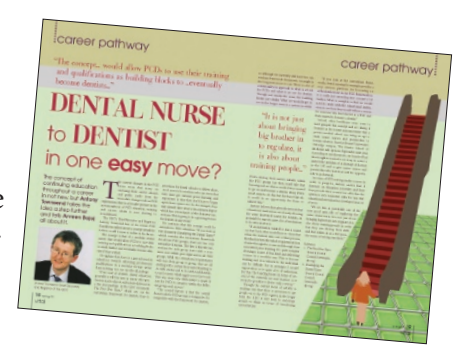
believe that all undergraduates should start as PCDs then climb the modular ladder. We would then perhaps have more rounded dental professionals who fully accept the concept of team work.

I chose a career in dentistry because I am passionate about teeth and after nine years of training I am still as dedicated as when I started my dental career. Let's hope we can learn from this experience and allow all PCDs to climb to the top of the career ladder.

Dave Martin,

Dental Technician, Dental Hygienist, Dental Therapist, Derbyshire 


\section{Increase income and confidence,}

\section{work closer with your technician}

The spread of Vital to dental professionals involved in patient treatment is going down well with most groups involved "in-house". But I am a little concerned that technicians working away from practices are not responding as positively as hoped. I would like to appeal to those essential members of the dental team, to wake up and smell the coffee.

Nobody is naïve enough to imagine that the relationships will change overnight but they will never change unless the technical specialist represents himself as a committed member of the patient-care team.

It is of course easier to ignore this scenario but the communication gap is increasing daily and is already of Grand-Canyon proportions. Relationships are already breaking down because the technician and clinician are not speaking the same language.

This is an intolerable situation for the patient. The law says that the clinician has the right and responsibility of prescription but are they all equipped to make the right decisions about the materials and choices which may suit the required situation?

Many are, but a growing number are not equipped to make judgements on the materials handled by technicians on a daily basis. Of course this involvement should be encouraged and supported by the clinician involved in the restorative procedure.
I would ask that the laboratory be encouraged and presented as a value added component for the patient and not just as the biggest part of the bill, which is pounced on by the annual audit! The cost of laboratory procedures should be measured by the delivery of the correct restoration to the patient.

Just like our clinical colleagues, all technicians are not of the same ability nor are they necessarily good communicators but they must be encouraged to become involved to a greater extent than many are at present.

My experience tells me that the involvement of the technician as a high profile member of the team always brings greater patient satisfaction and absolutely better outcomes for all concerned. So to my technical colleagues I would say don't just complain, make the effort to sell yourself and explain the vital role you play.

To my clinical colleagues I would say you are missing out on an opportunity to improve not just your restorative services but also the chance to gain greater confidence from your patients.

Let's work together for the improvement in our daily lives and for the higher standard that will undoubtedly be available to our patients.

Larry Browne,

Restorative Technician, Vital Editorial Board
SEND YOUR LETTERS to: the Editor, Vital Magazine, BDJ, Nature Publishing Group, 4-6 Crinan Street, London NI 9XW or email vitaleditorial@nature.com. Authors must sign the letter, which may be edited for reasons of space.

Our star letter writer will receive a Sonnet Black Laque fountain pen (£90 RRP) courtesy of Parker Pens.

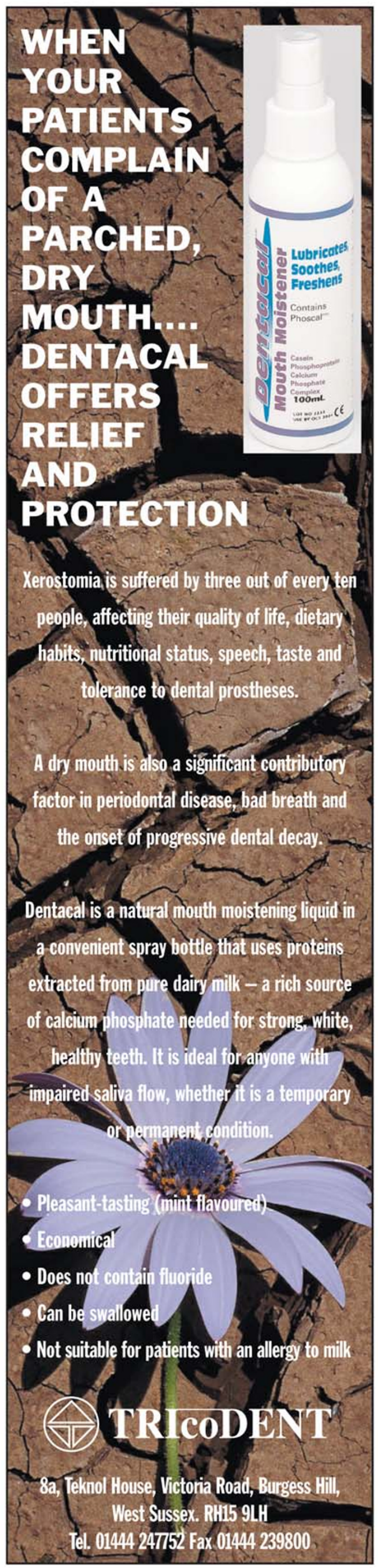

